\title{
Application of Combined Vessel Resection with Reconstruction in Complex Hepatobiliary and Pancreatic Space-Occupying Lesions
}

\author{
Zhixin Wang, Yanyan Zhou, Wei Chen, Kai Qu, Runchen Miao, Xinsen Xu, Ruitao Wang, \\ Chang Liu* \\ Department of Hepatobiliary Surgery, The First Affiliated Hospital, School of Medicine, Xi'an Jiaotong \\ University, Xi'an, China \\ Email: ${ }^{*}$ liuchangdoctor@163.com
}

Received 9 January 2015; accepted 24 January 2015; published 29 January 2015

Copyright (C) 2015 by authors and OALib.

This work is licensed under the Creative Commons Attribution International License (CC BY). http://creativecommons.org/licenses/by/4.0/

(c) (i) Open Access

\section{Abstract}

Aim: To explore the value and prospects of the combined vessel resection with reconstruction techniques in space-occupying lesion surgical treatment. Method: We collected and analyzed clinical data of patients received combined with vessel resection and reconstruction techniques surgery in department of hepatobiliary surgery in our hospital from May 2007 to November 2012, and concluded experience in application of vessel resection and artificial vessel reconstruction. Result: A total of 12 cases were collected. 9 of them were pancreatic lesion, and the other 3 were liver Echinococcus granulosus. The average operative time of 12 cases was $6.1 \pm 1.8 \mathrm{~h}$, and the average blood loss was $385 \pm 202 \mathrm{ml}$. The average intraoperative portal vein occlusion time was $31 \pm$ $13 \mathrm{~min} .1$ case suffered intraperitoneal hemorrhage within $48 \mathrm{~h}$ after surgery and other 2 suffered pancreatic fistula. All of them were treated and cured; there was no infection, thrombosis and other complications and perioperative deaths turned up before discharge. 4 cases of pancreatic cancer patients after 4, 9, 35 and 37 died of tumor recurrence and metastasis: 1 was lost and the rest are all alive. The follow-up time of these cases was 3 - 58 months with an average level of 22.4 months. Conclusion: Occupying lesions that cannot achieve traditional radical resection due to vessel invasion conventional radical resection may be cured by combining with vessel resection and reconstruction of the lesion resection surgery, and with good prospects worthy of promotion and application of clinic; this technique should be applied to lesion in hepatobiliary system.

\section{Keywords}

Combined Vessel Resection, Reconstruction, Space-Occupying Lesions

\footnotetext{
${ }^{*}$ Corresponding author.
}

How to cite this paper: Wang, Z.X., Zhou, Y.Y., Chen, W., Qu, K., Miao, R.C., Xu, X.S., Wang, R.T. and Liu, C. (2015) Application of Combined Vessel Resection with Reconstruction in Complex Hepatobiliary and Pancreatic Space-Occupying Lesions. Open Access Library Journal, 2: e1317. http://dx.doi.org/10.4236/oalib.1101317 
Subject Areas: Clinical Trials, Gastroenterology \& Hepatology, Surgery \& Surgical Specialties

\section{Introduction}

The hepatic portal and pancreas head are rich blood supply regions where vessels around are dense; many important vessels are distributed here, such as the portal vein (PV), superior or inferior mesenteric vein (SMV/ IMV), vena cava, etc. Thus, it is common that space-occupying lesions originating from these regions or nearby (such as liver, gallbladder or pancreas head cancer) are easy to invade vessels nearby; for instance, ashilar cholangiocarcinoma invades the main trunk or branch of portal vein; the hepatic cancer or echinococcosis invades one or more hepatic veins, etc. Once occurred, the success rate of integrity/radical resection was low, even regarded as surgical contraindication in the past.

Technique difficulty is the main reason handicapping the elevation of resection rate of complex hepatobiliary and pancreatic space-occupying lesions with vessel invasion. In addition, difficulties in management of some postoperative complications also contribute to the delay of the clinical application of radical resection for complex hepatobiliary and pancreatic space-occupying lesions therapy. In recent years, with the technological advancement in imageology, vessel surgery and materials in medicine, complex hepatobiliary and pancreatic space-occupying lesions resection combined with vessel resection and reconstruction, or other operations involved in vessel blocking or/and anastomosis have been becoming available gradually, and more and more related patients' outcome has been improved largely.

\section{Materials and Methods}

Information of patients admitted in department of hepatobiliary surgery in our hospital between March 2008 and November 2011, all of those underwent resection of hepatobiliary and pancreatic space-occupying lesions. Inclusion criteria: 1) space-occupying lesions with larger vessels invasion detected by preoperative (enhanced) CT and/or CTA examination; 2) cannot achieve radical resection by the conventional surgery; 3) receive resection combined with vessel resection and reconstruction, and the lesion, including the invaded vessel, was removed completely; 4) followed up by B-ultrasonic to observe reconstructed vessel blood flow and the presence of thrombosis at 1, 3, and 6 month after surgery. The information collected includes: preoperative examination results (imaging, biochemical test) operation note, postoperative examination (imaging, biochemical test, and pathology reports), etc. All patients followed up at 1, 3, and 6 month after surgery in hospital, and then in telephoned every 6 months until admitted again or death.

\section{Results}

The surgery that combined with vessel resection and reconstruction with artificial vessel for complex hepatobiliary and pancreatic cancer performed in 12 cases, including 5 males, 7 females, the youngest 29 years old and the oldest 65 years old. Preoperative diagnosis: pancreatic cancer six cases, liver hydatid disease three cases, Pancreatic pseudo papillary tumor 1 case, pancreatic neuroendocrine carcinoma 1 case and pancreatic carcinoma 1 case. Postoperative pathology confirmed: 3 were hepatic alveolar echinococcosis, 5 were invasive pancreatic adenocarcinoma, 1 was mucinous adenocarcinoma, 1 was invasive neuroendocrine carcinoma, and 2 were solid-pseudo papillary tumor (Table 1).

Surgical method: a case of extended right trisegmentectomy, remove of retrohepatic inferior vena cava and partial inferior vena cava, left hepatic vein was anastomosed end-to-end with suprahepatic inferior vena cava; a case of extended right trisegmentectomy, remove of retrohepatic inferior vena cava and partial inferior vena cava, portal vein (PV) reconstruction, and anastomosis of left hepatic vein and Su-prahepatic inferior vena cava with artificial vessel; a case of pancreatoduodenectomy combined with resection of partial Inferior mesenteric vein (SMV) and portal vein (PV); 4 cases of extended pancreatoduodenectomy with reconstruction of SMV and PV with artificial vessel (2 cases of 4, vessel anastomosis of spleen vein to artificial vessel was performed in an end-to-side fashion); a case of remove of tumor of body and tail of the pancreas combined with partial resection of SMV and PV, and reconstruction of SMV and PV with artificial vessel; 4 cases of remove of tumor of body or tail of the pancreas combined with resection of vessel, and SMV was anastomosed end-to-end with portal 
Table 1. Preoperative diagnosis and postoperative pathology.

\begin{tabular}{|c|c|c|c|c|}
\hline Case & Gender & Age & Preoperative diagnosis & Postoperative pathology \\
\hline 1 & Female & 38 & Hepatic alveolar echinococcosis & Hepatic alveolar echinococcosis \\
\hline 2 & Female & 53 & Carcinoma of head of pancreas & Invasive pancreatic head adenocarcinoma \\
\hline 3 & Female & 45 & Carcinoma of head of pancreas & Malignant solid pseudopapillary tumor of the pancreas \\
\hline 4 & Male & 65 & Carcinoma of head of pancreas & Invasive pancreatic head adenocarcinoma \\
\hline 5 & Male & 28 & Hepatic alveolar echinococcosis & Hepatic alveolar echinococcosis \\
\hline 6 & Male & 63 & Carcinoma of head of pancreas & Invasive adenocarcinoma at neck and body \\
\hline 7 & Female & 58 & Carcinoma of pancreatic body & Invasive pancreatic head adenocarcinoma \\
\hline 8 & Male & 48 & Carcinoma of head of pancreas & Invasive pancreatic head adenocarcinoma \\
\hline 9 & Female & 29 & $\begin{array}{l}\text { Pseudopapillary cystic neoplasm } \\
\text { of the pancreas(pncp) }\end{array}$ & Solid pseudopapillary tumor of the pancreas \\
\hline 10 & Male & 29 & $\begin{array}{l}\text { Pancreaticneuroendocrine } \\
\text { carcinoma(npnec) }\end{array}$ & $\begin{array}{l}\text { Invasive moderate differentiation neuroendocrine } \\
\text { carcinoma of head of pancreas }\end{array}$ \\
\hline 11 & Female & 56 & $\begin{array}{l}\text { Cystadenocarcinoma of body } \\
\text { and tail of pancreas }\end{array}$ & Hepatic alveolar echinococcosis \\
\hline 12 & Female & 63 & Hepatic alveolar echinococcosis & Moderate differentiation adenocarcinoma of the pancreas \\
\hline
\end{tabular}

vein; a case of extracorporeal right liver resection, reconstruction of inferior vena cava, autologous liver transplantation, and cholangioenterostomy (Table 2).

All of 12 cases were operated successfully, in addition to two cases of hemi-extracorporeal resection of hepatic hydatid undergo a long time $(13 \mathrm{~h})$, more bleeding $(2100 \mathrm{ml})$, the remaining 10 cases were completed within 5 - $8 \mathrm{~h}$, average $6.1 \pm 1.8 \mathrm{~h}$, blood loss was 150 - $600 \mathrm{ml}$, average $385 \pm 202 \mathrm{ml}$, intraoperative portal vein occlusion time 24 - 47 min, mean $31 \pm 13$ min. Postoperative management: In addition to conventional treatment after surgery, we must also pay attention to the following: 1) try to maintain a stable blood pressure, prevent blood pressure too low or too high; 2) reduce postoperative graft infection, prophylactic use of broad-spectrum antibiotics, the time is about 1 week; 3 ) at 1, 3, and 6 month after surgery etc.; 4) follow-up: regular B-revesselization to examine the artificial vessel blood flow and the presence of thrombosis at 1,3 , and 6 month after surgery. Since then, telephone follow-up performed every six months to understand the prognostic information (e.g. the survival, tumor recurrence or metastasis, etc.). In 1 cases of intra-abdominal hemorrhage occurred within $48 \mathrm{~h}$ after operation, the bleeding was stopped by a second operation. Pancreatic leakage occurred in 2 patients, both cured with sufficient drainage. No complications, such as infection, and no perioperative death were recorded. The follow-up period ranged from 3 to 58 months, with the mean of 44 months so far, and one patient was lost after 20-month follow-up. No complications were observed in the remaining 11 patients, four patients with pancreatic head carcinoma died of recurrence and the metastasis of cancer at 4, 9, 35, and 37 months postoperatively.

\section{Discussion}

In hepatobiliary system, the radical resection rate of lesions is rather low for the high frequency of accompanying important blood vessels invasion, such as portal vein (PV), superior mesenteric vein (SMV), hepatic veins (HVs), inferior vena cava, et al. In traditional surgical view, patients suffered from lesions have almost missed the opportunity of radical surgery for operation's huge trauma to the patients. In addition, the high rate of pathological positive surgical margin for technical reasons and the high rate of lymph node metastasis probably signify poor prognosis. So many believe that radical operation resection actually has no significance for patients with blood vessel invasion.

However with the development of operation technology, the pathological positive rate in surgical margin and lymph node metastasis have almost come to be no difference between patients with and without blood vessel invasion [1], which support the opinion that operation is a good choice to improve the prognosis for some patients suffered with space-occupying lesions with vessel invasion. Studies have also revealed that about $21 \%$ - 
Table 2. Invaded vessel and treatment.

\begin{tabular}{ccccc}
\hline Vessel invaded & Remove & Artificial vessel graft & Suture and repair & End to end anastomosis \\
\hline SMV & 4 & 4 & 4 & 1 \\
PV & 6 & 4 & 5 & 1 \\
Right (left) liver artery & 3 & - & - & - \\
Right (left) liver vein & 2 & - & - & 1 \\
Left renal vein (LRV) & - & - & 3 & 1 \\
Inferior vena cava (IVC) & - & - & - & - \\
Superior mesenteric artery (SMA) & 2 & - & 1 & 2 \\
Celiac trunk & 1 & - & 2 & - \\
Abdominal aorta & & &
\end{tabular}

43\% tumor specimens invading vessels, indistinguishable and inseparable to the naked eye, were inflammatory adhesion verified by postoperation pathology [1] [2]. Thus, giving up surgery for part of patients with blood vessel invasion may give a fundamental impact on patients' outcome, and it is meaningful for part of patients with space-occupying lesions to receive radical surgery. More and more patients suffered from malignant lesion with vessel invasion gain the chance of radical resection is largely benefited from the development of auxiliary inspection technique and equipment, such as multi-slice spiral CT, MRI, and so on. These progressions make it become a reality to make accurate assessment and sufficient preparation before operation. All of the 12 patients included received an abdominal CT/enhanced CT scanning before operation, and 6 cases out this cohort because of the unclear judgment gained from the above-mentioned preoperative examination were received a CTA examination further. The scanning results showed us compression, shift or intraluminal filling defect on the artery or vein nearby (PV, SMV, et al.), which reminded us the possibility of vessel invasion and the necessity to make well preoperative preparation for vessel resection and reconstruction.

However, resection of the lesion and vessels invaded may be inevitable for some patients for restriction of anatomy hurdles or space distance. Therefore, it comes out the requirements of the vessel material for reconstruction to overcome difficulty mentioned above. There are two main kinds of vessel for reconstruction, autologous and artificial vessel. The autologous vessel generally gained from the great saphenous vein or jugular vein of patient itself have favorable biocompatibility, but it is difficult to acquire and a long-term storage. Moreover, easy-damaged during acquisition, mismatches of diameter length, and other factors limit its popularization and application in operation. The artificial vessel made up with by Dacron was firstly used during dog's abdominal aorta reconstruction in 1952, which then applied in clinical in the next year. That was a milepost in the development of artificial vessel. And from then on, surgeons have saved a large number of lives by using various artificial vessels in the following decades.

The philosophy of lesions resection combined with vessel resection and reconstruction originates from curative surgery, beside the general principle for lesions resection, shorten the ischemia and congestion time of related organ and reduce complication [3]. According to reports and our experience, the following points below should be noticed: 1) Treatment for primary tumor. In the premise of resection of lesions as far as possible complete, shorten the time of vessel blocking during the operation. The distal vessels of lesion should be dissected firstly, then free and resect the lesion. 2) Treatment of portal vein. Tashiro et al. [4] believe that it is safe that the blocking time is limited within $60 \mathrm{~min}$, otherwise the shunt should be performed. To lesions with invaded vessels and hard to free, we can firstly free normal vessels around the lesion and performed lymphadenectomy before vessel blocking. It was helpful for shortening the blocking time of portal vein, and the vessel blocking time in our cohort was $32 \pm 13 \mathrm{~min}$ in average. 3) Treatment for vessel. According to the length of the two cut ends of vessels, the treatments for the two cut ends are different. Some believe that $3 \mathrm{~cm}$ is a suitable boundary length. The vessel resection and end-to-end anastomosis should be performed if the length of the two cut ends of vessel is less than $3 \mathrm{~cm}$, otherwise, the vessel transplantation (alternative channel) must be the choice [2]. When the length of vessel resected was too long, the falciform ligament could be cut off until the junction point of left hepatic veins and right hepatic veins joining into the inferior vena cava. The right below the diaph- 
ragm could be filled with gauze to push down the liver. Meanwhile, the root of mesostenium can be lifted in order to push the cut end of inferior mesenteric vein as far as up possible. We adopted a similar method, and regarded the length of $5 \mathrm{~cm}$ as the boundary point of different treatments [5]. Moreover, in some cases, such as vessels adhered to lesions tightly or vessels invaded by lesions directly, both the lesions and the local blood vessels invaded should be directly resected, and then reconstruct blood pathway by autologous or artificial vessel. The advantage of this processing is avoiding blood vessels rupture and bleeding, incompletely lesions resection, potential local recurrences which were caused by separating the lesion from the surface of the invaded vessels and reducing the time of blood vessels blocking and reconstruction during the operation. And the $2^{\#}$ and $9^{\#}$ cases of our cohort were treated by this method.

Hemorrhage and infection are common postoperative complications. One case of our cohort occurred abdominal hemorrhage within 48 hours after operation, caused by suturing vessels sparsely during vessel reconstruction and anastomosis by the emergency reoperation. And the bleeding was stopped by one needle of 5-0 Prolene line. Leakage of pancreatic juice appeared in two cases in one week after operation, and after drainage and the application of acid-suppressing drugs, they had been recovery when they were discharged. Other serious complications such as small bowel necrosis, severe abdominal infection did not occur in our cohort. The late complications in 30 days after operation such as intractable diarrhea, stiffness induced by irritable bowel tissue fibrosis, slowed intestinal motility and indigestion were not reported. Furthermore, it is very important to prevent thrombosis and embolism after operation involving vessel resection, anastomosis and reconstruction. Thrombosis, thrombosis, vessel occlusion and other related complications did not occur in our cohort. Besides the necessary treatments including anticoagulant and antithrombotic treatments, surgical operation is also very important. We injected heparin to intravessel (heparin dose is usually half the amount of the whole body, $0.5 \mathrm{mg} / \mathrm{kg}$ ) before blocking large vessels during surgery to prevent intravessel thrombosis. We used a 5-0 prolene lines to perform continuous exstrophy suture to ensure the vessel walls smooth. When completed the suture we drained out 50 $100 \mathrm{ml}$ of blood before opening the vessel to exhaust blood clots and air.

Statistics data shows that resection rate of conventional pancreaticoduodenectomy was only $10 \%$ to $20 \%$, and the median overall survival time was 12 - 16 months after operation and the 5-year survival rate was only about $5 \%$ [6]. Combining with vessel resection and reconstruction, especially radical resection of pancreatic cancer combined with artificial vessel reconstruction has been carried out and reported at Fudan University, Central South University, Nanjing Medical University and other larger medical centers [5] [7]-[15]. The resection rate of combined with vessel resection was up to $47 \%$, operative mortality rate was $<5 \%$ [16] [17] and 5-year survival rate was up to $20 \%$ [18]. There are 9 cases of pancreatic cancer patients in our cohort all cured and the perioperative mortality rate was $0 \% .4$ cases with pancreatic cancer died of malignancy recurrence and metastasis in 4,9 , 35, 37 months after operation, respectively. The remaining 5 cases are all alive so far. The postoperative survival time $\geq 20.2$ months, and the median survival time was longer compared with overall pancreatic cancer patients. Although the number of cancer patients adopted in our cohort was small, it could reflect that the pancreatic resection combined with vessel resection could improve the prognosis of patients. In addition, the cohort includes 3 cases of hepatic alveolar echinococcus eroded vessel heavily, and the conventional surgery cannot resect the lesions. The lesions were completely resected after vessel resection and reconstruction, and there were no serious postoperative complications after surgery. Hepatectomy of hepatocellular carcinoma combined with vessel resection and reconstruction has not been reported in China before, thus, this study is the first in our country. Although the alveolar hydatid disease of liver was a benign disease, its property of growth is similar to malignancy and there is a possibility of recurrence and metastasis. Thus it is well known as "worm malignancy". The 3 alveolar hydatid cases in our cohort have no recurrences and metastasis so far (Table 3). The follow-up time is short and the number of cases is small, so we cannot draw an exact conclusion that lesion resection combined with vessel resection and reconstruction contributes to the treatment of liver cancer, but it has a valuable reference and guiding significance for surgical treatment in the future.

\section{Conclusion}

With the continuous progress of imaging technology and operation, resection combined with vessel resection and reconstruction, more complex and risky than traditional common operation, must become a more popular choice in clinical practice. It not only reflects the progression of surgery technique, but also improves patients' outcome importantly for its providing more opportunity to cure or relieve symptoms of patients with occupying 
Table 3. Surgical method and prognostic conditions.

\begin{tabular}{|c|c|c|c|c|}
\hline Case & Operation & Complications & $\begin{array}{l}\text { Follow-up time } \\
\text { (months) }\end{array}$ & Outcome \\
\hline 1 & hepatic left lobectomy & - & 58 & Survival \\
\hline 2 & $\begin{array}{l}\text { Pancreaticoduodenectomy + partial resection and } \\
\text { reconstruction of portal vein }(\mathrm{PV})\end{array}$ & Pancreatic fistula & 9 & Death \\
\hline 3 & $\begin{array}{l}\text { Pancreaticoduodenectomy + resection and reconstruction of partial } \\
\text { portal vein }(\mathrm{PV})+\text { radio frequency ablation for hepatic metastasis }\end{array}$ & - & 35 & Death \\
\hline 4 & $\begin{array}{l}\text { Pancreaticoduodenectomy + resection and reconstruction } \\
\text { right hepatic artery and partial portal vein (PV) }\end{array}$ & - & 4 & Death \\
\hline 5 & $\begin{array}{l}\text { Right trisegmentectomy + retrohepatic inferior vena cava } \\
\text { resectionand distal closure, end-to-end anastomosis of left } \\
\text { hepatic vein and Su-prahepatic inferior vena cava }\end{array}$ & - & 26 & Survival \\
\hline 6 & $\begin{array}{l}\text { Pancreaticoduodenectomy + resection and reconstruction } \\
\text { of partial SMV with artificial vessle }\end{array}$ & - & 37 & Death \\
\hline 7 & $\begin{array}{l}\text { Pancreatic resection + splenectomy resection and } \\
\text { reconstruction of partial PV and SMV + cholangioenterostomy }\end{array}$ & - & 20 & Lost \\
\hline 8 & $\begin{array}{l}\text { Pancreaticoduodenectomy combined with portal vein } \\
\text { remove andreconstruction }\end{array}$ & $\begin{array}{l}\text { Abdominal } \\
\text { hemorrhage }\end{array}$ & 25 & Survival \\
\hline 9 & $\begin{array}{c}\text { Pancreaticoduodenectomy + splenectomy + reconstruction } \\
\text { of portal vein }(\mathrm{PV}) \text { with artificial vessel }\end{array}$ & - & 24 & Survival \\
\hline 10 & $\begin{array}{c}\text { Pancreaticoduodenectomy + cholecyst resection + inferior } \\
\text { vena cava artificial vessel bypass }\end{array}$ & - & 19 & Survival \\
\hline 11 & $\begin{array}{l}\text { Pancreatic body and tail resection + splenectomy + resection of } \\
\text { hepatic metastasis + partial resection and reconstruction of portal } \\
\text { vein }(\mathrm{PV})+\text { resection of common hepatic artery and celiac trunk }\end{array}$ & Pancreatic fistula & 9 & Survival \\
\hline 12 & $\begin{array}{l}\text { Extracorporeal right liver resection + reconstruction of } \\
\text { inferior vena cava + autologous liver transplantation }\end{array}$ & - & 3 & Survival \\
\hline
\end{tabular}

malignancy. Thus, resection combined with vessel resection and reconstruction is worthy of clinical application.

\section{Acknowledgements}

This work was financially supported by Program for Innovative Research Team of Shaanxi Province (2013KCJ-23).

\section{References}

[1] Aramaki, M., Matsumoto, T., Etoh, T., et al. (2003) Clinical Significance of Combined Pancreas and Portal Vein Resection in Surgery for Pancreatic Adenocarcinoma. Hepato-Gastroenterology, 50, 263.

[2] Han, S.S., Park, S.J., Kim, S.H., et al. (2012) Clinical Significance of Portal-Superior Mesenteric Vein Resection in Pancreatoduodenectomy for Pancreatic Head Cancer. Pancreas, 41, 102. http://dx.doi.org/10.1097/MPA.0b013e318221c595

[3] Cusack Jr., J.C., Fuhrman, G.M., Lee, J.E., et al. (1994) Managing Unsuspected Tumor Invasion of the Superior Mesenteric-Portal Venous Confluence during Pancreaticoduodenectomy. American Journal of Surgery, 68, 352. http://dx.doi.org/10.1016/S0002-9610(05)80164-3

[4] Tashiro, S., Uchino, R., Hiraoka, T., et al. (1991) Surgical Indication and Significance of Portal Vein Resection in Biliary and Pancreatic Cancer. Surgery, 109, 481-487.

[5] Yang, W.P., Peng, C.H., Shao, T.L., et al. (2006) On Pancreatoduodenectomy in Combination with Vascular Resection. Chinese Journal of Hepatobiliary Surgery, 12, 742-742.

[6] Ahmad, N.A., Lewis, J.D., Ginsberg, G.G., et al. (2001) Long Term Survival after Pancreatic Resection for Pancreatic Adenocarcinoma. The American Journal of Gastroenterology, 96, 2609-2615. http://dx.doi.org/10.1111/j.1572-0241.2001.04123.x

[7] Yang, J., Chen, F.Z., Guo, X.F., Cai, M.Z., et al. (2001) Problems of Vascular Reconstruction Combined Pancreatoduoenectomy (Report of 45 Cases). Clinical Medical Journal of China, 8, 609-611. 
[8] Chen, F.Z., Ge, X.H., Zhao, D.J., et al. (2002) Problems of Vascular Reconstruction Combined Pancreatoduodenectomy: Report of 42 Cases. Chinese Journal of Practical Surgery, 22, 273.

[9] Shu, C., Zhou, Y.D., Lu, X.S., et al. (2004) Surgical Treatment of Major Vessels Invaded by Tumor. Chinese Journal of General Surgery, 13, 523-526.

[10] Ge, X.-H., Chen, F.-Z., Zhao, D.-J., et al. (2004) Combined Pancreatoduodenectomy and Vascular Reconstruction in the Treatment of Pancreatic Head Malignant Tumors. Chinese Journal of Hepatobiliary Surgery, 19, 37-39.

[11] Li, B., Chen, F.-Z., Ge, X.-H., et al. (2005) Vascular Reconstruction Combined Pancreatoduodenectomy to Treat the Malignant Tumor at the Caput Pancreatis. Journal of Hepatopancreatobiliary Surgery, 17, 23-25.

[12] Gao, X., Zhang, J.P., Wang, K.J., et al. (2009) Resection Combined with Vascular Reconstruction for Pancreatic Cancer. Journal of Clinical Medicine in Practice, 13, 108-108.

[13] Xiang, H.S., Li, Y., Fen, Z.Y., et al. (2010) Clinical Analyse of Modified Whipple Surgical and Interventional Chemotherapy in Advanced Pancreatic Cancer Radical Mastectomy. Chinese Journal of Clinicians (Electronic Edition), 1, 019.

[14] Zhang, H.Y., Xiao, Z.Y., Ye, H., et al. (2012) Combined Revascularization for Superior Mesenteric Vein Invaded Pancreaticoduodenectomy (Case Report and Literature Review). Journal of Digestive Oncology (Electronic Version), 4.

[15] Guan, X.-Q., Chen, Y., Gu, S.-C., et al. (2012) Feasibility Study on Clinical Application of Hepatic Artery, Proper Hepatic Artery, and Internal Iliac Vein Resection and Reconstruction in Extended Pancreaticoduodenectomy. Chinese Journal of Bases and Clinics in General Surgery, 19.

[16] Howard, J.M. (1999) Development and Progress in Resective Surgery for Pancreatic Cancer. World Journal of Surgery, 23, 901-906. http://dx.doi.org/10.1007/s002689900597

[17] Takahashi, S., Ogata, Y. and Tsuzuki, T. (2005) Combined Resection of the Pancreas and Portal Vein for Pancreatic Cancer. British Journal of Surgery, 81, 1190-1193. http://dx.doi.org/10.1002/bjs.1800810837

[18] Peng, S.Y. and Qian, H.R. (2006) The Complicated Pancreaticoduodenectomy. Chinese Journal of Bases and Clinics in General Surgery, 13, 496-497. 
Scientific Research Publishing (SCIRP) is one of the largest Open Access journal publishers. It is currently publishing more than 200 open access, online, peer-reviewed journals covering a wide range of academic disciplines. SCIRP serves the worldwide academic communities and contributes to the progress and application of science with its publication.

Other selected journals from SCIRP are listed as below. Submit your manuscript to us via either submit@scirp.org or Online Submission Portal.
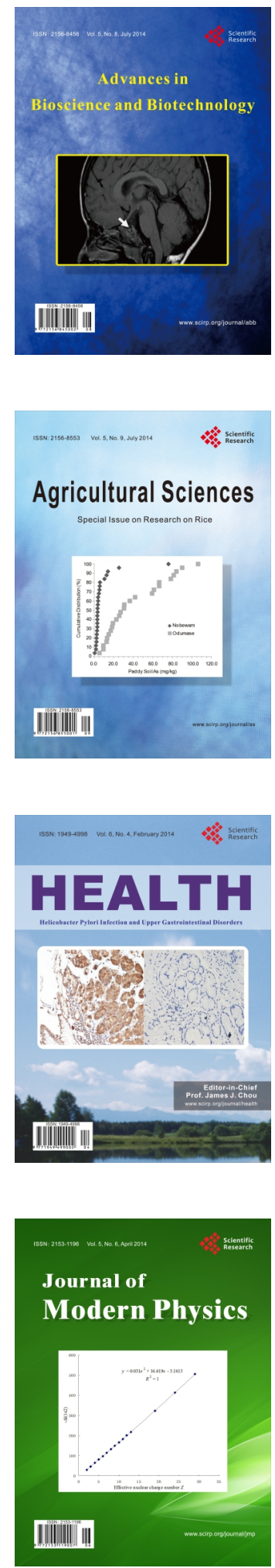
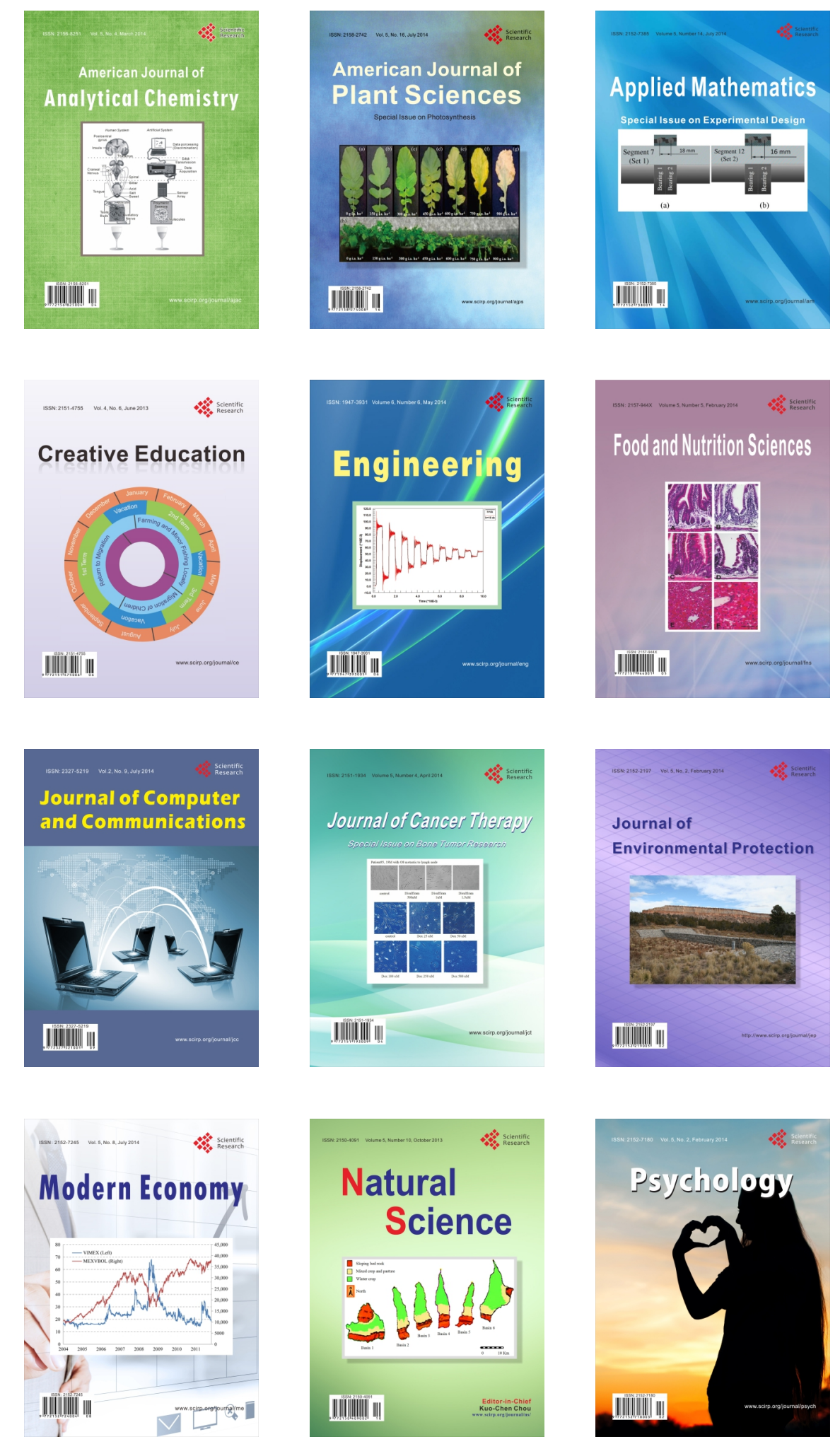